\title{
Characterization of Non-Polar ZnO Layers with Positron Annihilation Spectroscopy
}

\author{
A. Zubiaga ${ }^{a}$, F. Tuomisto ${ }^{a}$, J. ZÚNĩiga-PÉReZ $^{b}$ \\ AND V. MUÑOZ-SAN JosÉc \\ ${ }^{a}$ Department of Engineering Physics \\ Helsinki University of Technology \\ P.O. Box 1100, 02015 TKK Espoo, Finland \\ ${ }^{b}$ Centre de Recherche sur l'Hétéro-Epitaxie et ses Applications (CRHEA) \\ Centre National de la Recherche Scientifique (CNRS), 06560 Valbonne, France \\ ${ }^{c}$ Universitat de València, Departamento de Fisica Aplicada i Electromagnetisme \\ Dr. Moliner 50, 46100 Burjassot, Valencia, Spain
}

\begin{abstract}
We applied positron annihilation spectroscopy to study the effect of growth polarity on the vacancy defects in $\mathrm{ZnO}$ grown by metal-organic vapor phase deposition on sapphire. Both $c$-plane and $a$-plane $\mathrm{ZnO}$ layers were measured, and $\mathrm{Zn}$ vacancies were identified as the dominant defects detected by positrons. The results are qualitatively similar to those of earlier experiments in GaN. The $\mathrm{Zn}$ vacancy concentration decreases in $c$-plane $\mathrm{ZnO}$ by almost one order of magnitude (from high $10^{17} \mathrm{~cm}^{-3}$ to low $10^{17} \mathrm{~cm}^{-3}$ ) when the layer thickness is increased from 0.5 to $2 \mu \mathrm{m}$. Interestingly, in $a$-plane $\mathrm{ZnO}$ the $\mathrm{Zn}$ vacancy concentration is constant at a level of about $2 \times 10^{17} \mathrm{~cm}^{-3}$ in all the samples with thicknesses varying from 0.6 to $2.4 \mu \mathrm{m}$. The anisotropy of the Doppler broadening of the annihilation radiation parallel and perpendicular to the hexagonal $c$-axis was also measured.
\end{abstract}

PACS numbers: 61.72.uj, 68.55.Ln, 68.37.-d, 78.70.Bj

\section{Introduction}

The fabrication of photonic devices of ultraviolet wavelength range and high frequency electronic devices requires semiconductors with a wide band gap. The physical and chemical properties of $\mathrm{ZnO}$ make it a promising candidate for optoelectronic applications in the blue-UV range [1]. It is a semiconducting compound of the II-VI family with a direct wide band gap (3.37 eV at room temperature) 
and a large exciton binding energy $(60 \mathrm{meV})$. This makes it a promising candidate as a UV emitter at room temperature.

Due to its relative low cost, its availability in large area wafers, and its transparency up to about $6 \mu \mathrm{m}$ light wavelength, sapphire is a potential substrate for mass production of $\mathrm{ZnO}$ layers. Most usual substrate is $c\left(\begin{array}{llll}0 & 0 & 0 & 1\end{array}\right)$ sapphire but it induces the growth of polar $\mathrm{ZnO}$ layers. $\mathrm{Zn}$ and $\mathrm{O}$ polarity surfaces can spontaneously get charged [2]. The subsequent electric field can affect the growth process inducing the creation of higher concentration of defects. To overcome this problem non-polar $\mathrm{ZnO}$ layers are grown over $r$ sapphire $\left(\begin{array}{llll}1 & -1 & 0 & 2\end{array}\right)$ which does not have polar surfaces.

The growth polarity has been shown to have a dramatic effect on the in-grown point defects in $\mathrm{GaN}[3,4]$. In $c$-plane GaN, the incorporation of impurities and formation of native defects are significantly enhanced in growth in the $\mathrm{N}$ polar (as opposed to Ga polar) direction and their distribution of impurities and native defects is highly nonuniform as a function of the distance from the GaN/sapphire interface. On the other hand, in non-polar $a$-plane GaN the defect distributions are flat and the concentrations are similar to $\mathrm{N}$ polar GaN.

Positron annihilation spectroscopy (PAS) is a useful tool for studying the $\mathrm{Zn}$ vacancy in $\mathrm{ZnO}$. Positrons mainly get trapped at negative or neutral vacancy defects at room temperature. Zn vacancy can be negatively charged when it appears isolated from other defects [5] or in neutral state when it forms a complex with other non-open volume defect [6].

\section{Experimental}

We have applied positron annihilation spectroscopy to study point defects in $\mathrm{ZnO}$ layers formed during the growth process. $\mathrm{ZnO}$ layers have been grown by metal-organic chemical vapor deposition (MOCVD) on sapphire substrates oriented in $r$ plane $\left(\begin{array}{llll}1 & -1 & 0 & 2\end{array}\right)$. Layers were grown at atmospheric pressure in a horizontal MOCVD reactor (MR Semicon 102) with a two-inlet configuration, avoiding pre-reactions in the gas phase between the zinc and oxygen precursors: dimethylzinc-triethylamine (DMZn-TEN) and tertiary-butanol (t-butanol). The deposition time ranged between $45 \mathrm{~min}$ and $180 \mathrm{~min}$. For more details see Ref. [7]. An undoped single crystal from Eagle Picher, without positron traps at room temperature and with a positron lifetime of $170 \mathrm{ps}$, was used as reference. Results have been compared to $\mathrm{ZnO}$ layers grown over $c$-face of sapphire $\left(\begin{array}{llll}0 & 0 & 0 & 1\end{array}\right)$ that were reported in a previous work [8].

The experiments were performed at room temperature with a monoenergetic slow positron beam in the $0.5-38 \mathrm{keV}$ range. The Doppler broadening of the annihilation radiation was measured using a Ge detector with an energy resolution of $1.24 \mathrm{keV}$ at $511 \mathrm{keV}$. In each spectrum $1.5-2.0 \times 10^{6}$ counts were collected. The annihilation peak was analyzed using the conventional shape parameters: $S$ and $W$ parameters. The energy windows used were $\left|E_{\gamma}-511 \mathrm{keV}\right|<0.8 \mathrm{keV}$ 
for the central $S$ parameter and $2.9 \mathrm{keV}<\left|E_{\gamma}-511 \mathrm{keV}\right|<7.4 \mathrm{keV}$ for the wing $W$ parameter. $S$ and $W$ parameters are related to the annihilation with valence and core electrons, respectively. $S$ and $W$ parameters were measured in two perpendicular directions rotating the layer in the surface plane by $90^{\circ}$.

\section{Results and discussion}

Figure 1 shows the normalized $S$ and $W$ parameters measured in the $\mathrm{ZnO}$ layers grown over $r$-sapphire. They have been measured perpendicular to the $c$ hexagonal axis. $S$ and $W$ parameters of the bulk state measured in the direction perpendicular to the hexagonal axis have been used as reference for the normalized values. The graph in the left shows the evolution of the parameters versus the implantation energy of positrons for two layers and a reference sample. The reference sample has no positron traps at room temperature and all positrons
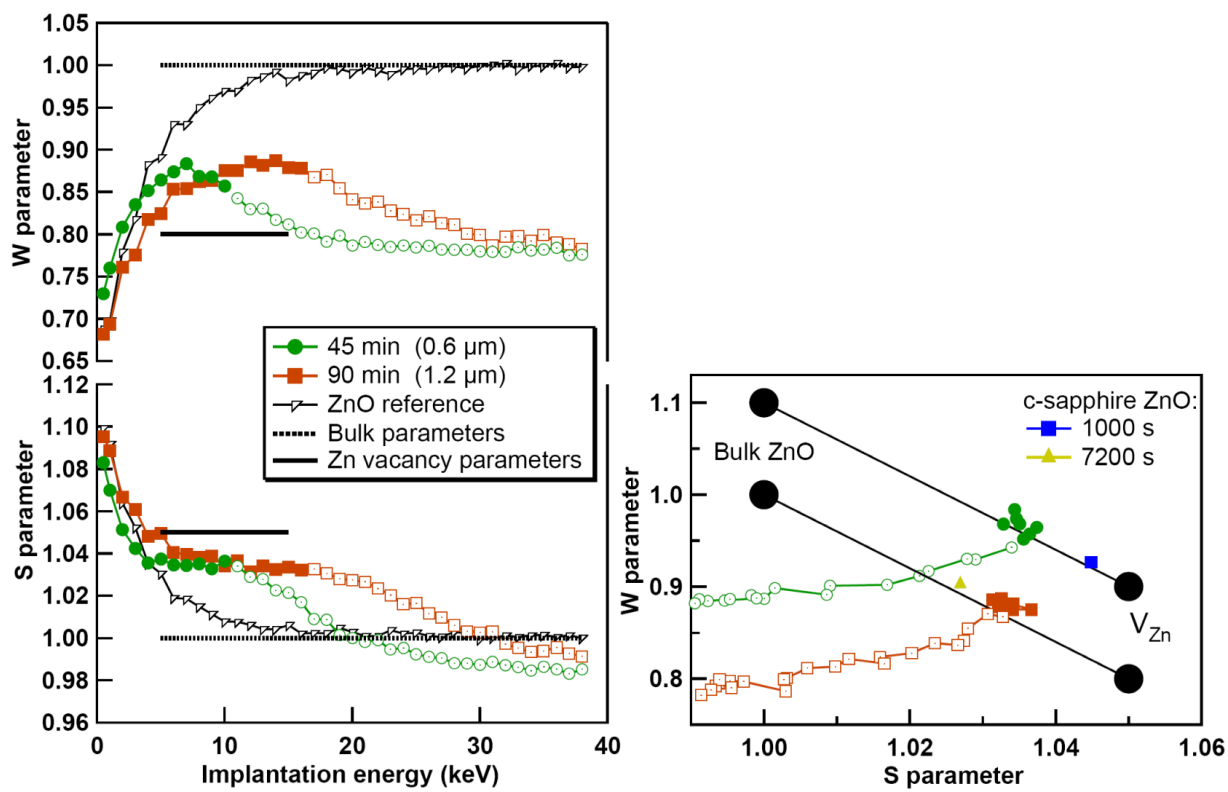

Fig. 1. In the left graph, the $S$ and $W$ parameters of non-polar $r$-ZnO layers versus the implantation energy are presented. The $W$ and $S$ parameters of a $\mathrm{ZnO}$ reference sample versus the implantation energy are also shown for comparison. Its value for high implantation energies (30-40 keV) shows the values of the $S$ and $W$ parameters for annihilation in bulk $\mathrm{ZnO}$. On the right, the $W$ parameter versus the $S$ parameter is plotted. The $W$ versus $S$ plot also shows the Zn vacancy value. Full symbols represent the points corresponding to energy values at which positrons annihilate only in the thin layer without any influence from the surface or the substrate. The cusp values of polar $\mathrm{ZnO}$ layers are also shown, for comparison. The points corresponding to the thinner non-polar and polar layers are shifted for better visualization. 
annihilate in delocalized states. Its behavior is typical of a sample with only oneannihilation state in the bulk. Positrons of low implantation energy annihilate in the surface and at high implantation energies all positrons annihilate in the bulk of the material, in delocalized states. In $\mathrm{ZnO}$ thin layers, positrons also annihilate at surface states at low implantation energies, at intermediate implantation energies (5-15 keV) annihilate inside the layer and at high implantation energies $(30-40 \mathrm{keV})$ positrons annihilate in the sapphire substrate. Between 15 and $30 \mathrm{keV}$ positrons annihilate both in the layer and the substrate, with a higher fraction of positrons annihilating in the substrate as the implantation energy increases.

$W$ parameter versus $S$ parameter graph (Fig. 1, right) shows that the points corresponding to positrons annihilating inside the layer lie within the $\mathrm{Zn}$ vacancy line. The $\mathrm{Zn}$ vacancy line is defined by the parameters of the bulk $\mathrm{ZnO}$ in one end and the parameters of the $\mathrm{Zn}$ vacancy on the other end. It shows that $\mathrm{Zn}$ vacancy related defects are the main defect trapping positrons inside the layer. Positrons start to annihilate in the sapphire substrate at higher implantation energy in the thicker sample $(16 \mathrm{keV})$ than in the thinner sample $(10 \mathrm{keV})$. It is consistent with a Makhovian implantation profile

$$
l(z)=\frac{2 z}{z_{0}^{2}} \exp \left(-\left(z / z_{0}\right)^{2}\right),
$$

where $z_{0}=1 / 0.886 \times 40 / \rho\left[\mathrm{g} / \mathrm{cm}^{3}\right] E[\mathrm{keV}]^{1.6}, \rho$ stands for the mass density and $E$ is the implantation energy.

Figure 1 also shows the $W / S$ cusp parameters for two $\mathrm{ZnO}$ layers grown over the $c$ face of sapphire.

\section{TABLE I}

Defect concentration and thickness of non-polar $r$-ZnO layers.

\begin{tabular}{c|c|c}
\hline \hline Growth time $[\mathrm{s}]$ & Defect concentration $\left[10^{17} \mathrm{~cm}^{-3}\right]$ & Thickness $[\mu \mathrm{m}]$ \\
\hline 2700 & $1.7(3)$ & $0.6(1)$ \\
5400 & $1.6(3)$ & $1.2(1)$ \\
8100 & $1.6(3)$ & $1.7(1)$
\end{tabular}

It is possible to estimate the layer thickness as twice the mean implantation depth $\left(\langle z\rangle=0.886 z_{0}\right)$ of positrons with the highest implantation energy possible without reaching the substrate (abandon point). Results are shown in Table I for non-polar layers.

\subsection{Vacancy profile}

The $S$ parameters within the $\mathrm{ZnO}$ layers, ranging between 1.030 and 1.307, are uniform, and they do not present the strong depth profile observed in polar samples [8]. The $W / S$ curve shows a cusp corresponding to positrons annihilating in the half of the sample in contact with the surface. The concentration of $\mathrm{Zn}$ 
vacancies is minimal there and $S(W)$ parameter has the smaller (larger) values. Positrons annihilating inside the layer with larger implantation energy than the cusp value have similar or slightly larger (smaller) $S(W)$ parameters. It is an indication that the concentration of $\mathrm{Zn}$ vacancies increases slightly near the interface between the $\mathrm{ZnO}$ layer and the sapphire substrate.

In the $W / S$ plot of the thinnest sample (45 min) the cusp values and the abandon point (see above or Ref. [9] for its definition) are slightly separated due to a decrease in the $W$ parameter. In thicker samples the cusp value and the abandon point are the same. The increase is very small when compared to polar layers grown over $c$-sapphire (see Ref. [8]) which indicates that the content of vacancies is more homogeneous along the growth direction. On the other side, in polar samples of comparable thickness cusp values of thick samples $(0.88 \mu \mathrm{m})$ has clearly smaller (larger) $S(W)$ parameters than thin samples $(0.48 \mu \mathrm{m})$ [8], while in non-polar samples there is not a big influence.

The concentration of $\mathrm{Zn}$ vacancies has been calculated from the $S$ parameter of the cusp measured at the perpendicular direction. The conventional trapping model gives the vacancy concentration

$$
\left[\mathrm{V}_{\mathrm{Zn}}\right]=\frac{N_{\mathrm{at}}}{\mu_{\mathrm{Zn}} \tau_{\mathrm{b}}}\left(\frac{S-S_{\mathrm{b}}}{S_{\mathrm{Zn}}-S}\right),
$$

where $N_{\text {at }}=8.22 \times 10^{22} \mathrm{~cm}^{-3}$ is the atomic density of $\mathrm{ZnO}, S_{\mathrm{b}}=1$ the $S$ parameter of the bulk and $S_{\mathrm{Zn}}=1.05$ the $S$ parameter of the $\mathrm{Zn}$ vacancy, respectively. $\mu_{\mathrm{Zn}}=3 \times 10^{15} \mathrm{~s}^{-1}$ is the trapping coefficient of the defect. There has been used the value of the Ga vacancy in GaN that was measured in Ref. [10]. Concentration values are shown in Table I.

Monoenergetic positrons get implanted according to the Makhovian profile of Eq. (1) and their implantation probability extends from the surface up to twice the mean implantation depth with a maximum probability around the mean implantation depth. For the cusp positions, positrons annihilate in the first half of the sample closer to the surface and they have maximum implantation probability at around one quarter of the thickness close to the surface. At higher depths inside the layers, closer to the interface with the substrate, the Zn vacancy concentration increases. This increase is observed as a larger (smaller) $S$ parameter ( $W$ parameter) near the abandon point.

In the thinnest layers the cusp position measures the $\mathrm{Zn}$ vacancies that are at distance from the interface equal or higher than $0.3 \mu \mathrm{m}$. In the thickest layers, on the other side, this distance is equal or higher to $0.6 \mu \mathrm{m}$. The influence of the interface at the cusp position is bigger in the thinnest layers. It can be seen that this influence is much smaller in non-polar layers (the $\mathrm{Zn}$ vacancy concentration remains almost constant at $1.6 \times 10^{17} \mathrm{~cm}^{-3}$ ) but it induces a clear increase in the defect concentration in polar layers (from $1.6 \times 10^{17} \mathrm{~cm}^{-3}$ to $6 \times 10^{17} \mathrm{~cm}^{-3}$ ) [8]. It is remarkable that in both types of samples the thicker sample has similar concentration of defects. 
In polar layers the $c$-hexagonal axis of the $\mathrm{ZnO}$ layer is parallel to the growth direction. The anisotropy characteristic of the $\mathrm{ZnO}$ wurtzite structure allows to create surface charges during the growth process. The electric field induced by this charge can be particularly intense in the first stage of the growth when the $\mathrm{ZnO}$ layer is very thin. It perturbs the deposition of new atoms and introduces a big amount of defects. As the sample grows thicker and the interface to the surface distance is larger, the influence of the surface charges becomes weaker and the growth process can occur without further perturbations. As a result, near the interface a higher concentration of defects is induced. On the other side, in non-polar layers the $c$-hexagonal axis lies within the layer surface. Then, the surface has not a clear polarity and there are no electric fields induced during the growth process. It allows a uniform growth of the layer at earlier stages. Closer than $0.3 \mu \mathrm{m}$ to the interface, a small increase in defects is still observed (see in Fig. 1 the small increase (decrease) in $S$ parameter ( $W$ parameter) in the sample of $0.6 \mu \mathrm{m}$ ) because there are still stresses due to the lattice mismatch between the $\mathrm{ZnO}$ layer and the substrate.

A similar behavior has been measured for the Ga vacancy in GaN [4]. The structural and point defect distributions of hydride vapor phase epitaxial (HVPE) GaN layer grown in the [11-20] a direction on (1-102) $r$-plane sapphire with metal-organic vapor phase deposited $a$-GaN template. Positron annihilation spectroscopy was used to measure the concentration of $\mathrm{Ga}$ vacancies. It is constant as a function of thickness, contrary to the non-uniform defect distribution observed in $\mathrm{GaN}$ grown along the [0001] direction.

\subsection{Hexagonal anisotropy}

Crystal structure of $\mathrm{ZnO}$ is wurtzite with hexagonal symmetry. This structure is not isotropic when the hexagonal axis is compared to any perpendicular axis, and it is isotropic within the plane perpendicular to the hexagonal axis. The electronic density and the moment distribution have the same symmetry properties. More precisely the momentum distribution along the hexagonal axis should be different to the moment measured in any perpendicular direction. In non-polar $\mathrm{ZnO}$ layers the hexagonal axis lies in the layer plane and its perpendicular direction, lying also in the surface plane, is non-equivalent. It allows to measure the electronic moment distribution in both directions.

The electronic moment distribution in the bulk state and the $\mathrm{Zn}$ vacancy have an anisotropy in the hexagonal direction but the amount of the anisotropy is different in both states. The difference in the momentum distribution will be reflected in the Doppler broadening of the $\gamma$ photon energy broadening measured in mentioned two perpendicular directions and their $S$ and $W$ parameters will be different.

The Doppler broadening in both directions has been compared measuring the annihilation radiation emitted parallel and perpendicular to the $c$ hexagonal axis. The values corresponding to the cusp positions are shown in Table II. 
The similarity of the values measured in all layers is remarkable. They are $1.026-1.028$ for the direction parallel to the $c$ hexagonal axis and 1.030-1.035 for the perpendicular direction. Thinnest samples have slightly larger $S$ parameters in parallel and perpendicular directions. The increase in the $S$ parameter is more marked in the perpendicular direction.

\section{TABLE II}

$S$ parameter of $\mathrm{ZnO}$ layers measured perpendicular and parallel to the measurement direction.

\begin{tabular}{c|c|c}
\hline \hline Growth time $[\mathrm{s}]$ & Parallel & Perpendicular \\
\hline 2700 & 1.0275 & 1.0351 \\
5400 & 1.0278 & 1.0325 \\
8100 & 1.0273 & 1.0304 \\
10800 & 1.0264 & 1.0301
\end{tabular}

There is a slightly higher contribution from low momentum valence electrons in the perpendicular direction due to the decreased overlapping with core electrons in that direction. The empty volume of the vacancy is slightly higher in that direction. The orientation perpendicular to the hexagonal axis is more suitable for measuring the $\mathrm{Zn}$ vacancy concentration than the parallel one.

\section{Conclusions}

Non-polar $\mathrm{ZnO}$ layers grown over $r$-sapphire have been measured using Doppler broadening positron annihilation spectroscopy in a slow positron beam. The $\mathrm{Zn}$ vacancy has been found to be the main positron trap in good accordance with previous experiments. Its concentration has been measured and it has been found that it is equal to $1.6 \times 10^{17} \mathrm{~cm}^{-3}$ in all non-polar layers. The uniformity of the samples thicker than $0.3 \mu \mathrm{m}$ is in contrast to the result in polar layers where there is a clear increase in $\mathrm{Zn}$ vacancy concentration even at $0.3-0.4 \mu \mathrm{m}$ from the interface compared to distance from the interface of $0.6 \mu \mathrm{m}$. Similar behavior has been previously observed in $c$-plane (polar) and $a$-plane (non-polar) HVPE GaN. It has been observed that $1.6 \times 10^{17} \mathrm{~cm}^{-3}$ is a typical value of $\mathrm{ZnO}$ thin layers grown over sapphire using MOCVD.

\section{Acknowledgments}

This work was supported by the Academy of Finland and the "Department of Education, Universities and Research" of the Basque Government through the "Program for the Formation of Researchers". We thank Mr. Ander Villate for the assistance in the positron experiments. 


\section{References}

[1] U. Özgur, Y. Alivov, C. Liu, A. Teke, M. Reshchikov, S. Dogan, V. Avrutin, S.J. Cho, H. Morko, J. Appl. Phys. 98, 041301 (2005).

[2] C. Morhain, T. Bretagnon, P. Lefebvre, X. Tang, P. Valvin, T. Guillet, B. Gil, T. Taliercio, M. Teisseire-Doninelli, B. Vinter, C. Deparis, Phys. Rev. B 72, 241305(R) (2005).

[3] F. Tuomisto, K. Saarinen, B. Lucznik, I. Grzegory, H. Teisseyre, T. Suski, S. Porowski, P.R. Hageman, J. Likonen, Appl. Phys. Lett. 86, 031915 (2005).

[4] F. Tuomisto, T. Paskova, R. Kröger, S. Figge, D. Hommel, B. Monemar, Appl. Phys. Lett. 90, 121915 (2007).

[5] F. Tuomisto, V. Ranki, K. Saarinen, D.C. Look, Phys. Rev. Lett. 91, 205502 (2003).

[6] F. Tuomisto, K. Saarinen, K. Grasza, A. Mycielski, Phys. Status Solidi B 243, 794 (2006).

[7] C. Munuera, J. Zúñiga-Pérez, J.F. Rommeluere, V. Sallet, R. Triboulet, F. Soria, V. Muñoz-San José, C. Ocal, J. Cryst. Growth 264, 70 (2004).

[8] A. Zubiaga, F. Tuomisto, F. Plazaola, K. Saarinen, J.A. Garcia, J.F. Rommeluere, J. Zuñiga-Pérez, V. Muñoz-San José, Appl. Phys. Lett. 86, 042103 (2005).

[9] A. Zubiaga, J.A. García, F. Plazaola, F. Tuomisto, J. Zúñiga-Pérez, V. MuñozSan José, Phys. Rev. B 75, 205305 (2007).

[10] K. Saarinen, T. Suski, I. Grzegory, D.C. Look, Phys. Rev. B 64, 233201 (2001). 